\title{
Immobilization and experimental periodontitis in tibial anterior muscle of wistar rats
}

\author{
Maria Luiza Serradourada Wutzke', Marcela Aparecida Leite', Carlos Augusto Nassar', \\ Patrícia Oehlmeyer Nassar ${ }^{1}$, Rose Meire Costa Brancalhão ${ }^{1}$, Gladson Ricardo Flor Bertolini', \\ Lucinéia de Fátima Chasko Ribeiro' \\ 1 Universidade Estadual do Oeste do Paraná, Brazil
}

Serradourada Wutzke ML, Leite MA, Nassar CA, Nassar PO, Costa Brancalhão RM, Flor Bertolini GR, Lucinéia de Fátima Chasko Ribeiro. Immobilization and experimental periodontitis in tibial anterior muscle of wistar rats. J Pre-Clin Clin Res. 2019; 13(1): 1-4. doi: 10.26444/jpccr/104640

\begin{abstract}
Introduction and objective. Periodontal disease (PD) is a chronic inflammation, locally attacking the supporting tissues of the tooth; this inflammatory condition can affect other systems, such as muscle tissue, which is also susceptible to several types of injury such as caused by immobilization. The aim of this study was to assess whether periodontal disease potentiates the deleterious effects of immobilization of the anterior tibial muscle of Wistar rats.

Materials and method. Twenty animals were randomly assigned to four groups $(n=5)$ : Control Group (CG), Periodontal Disease Group (PDG), Immobilized Group (IG) and PD plus Immobilization Group (PDIG). PD was induced on day 1 of the experiment by placing a cotton thread in the first lower molars for 30 days, favouring the accumulation of bacterial plaque. On day 15 , the right pelvic limb was immobilized for 15 days. On day 30, the animals were euthanized and the tibialis anterior muscle was collected with further processing for histomorphometric analysis.

Results. In the cross-sectional area there were PDIG differences between CG and PDG. For the larger diameter, CG was higher than IG and PDIG. For the smaller diameter, PDIG presented a lower value among all the groups in the same way with the number of nuclei. For connective tissue, there was an increase in the amount between the CG and PDIG, IG and PDIG groups, as well as PDG and PDIG.

Conclusion. Periodontal disease potentiated the deleterious effects of immobilization on the anterior tibialis muscle.
\end{abstract}

\section{Key words}

atrophic muscular disorders; atrophy; inflammation; periodontitis; immobilization

\section{INTRODUCTION}

The oral cavity is the gateway to pathogenic microorganisms [1] and once installed these agents can lead to the development of diseases, such as periodontal disease, which is a chronic inflammation established by the permanence of biofilm in the gingival sulcus which begins to aggressively damage the tooth's supporting tissues. The destruction of toothsupporting tissues may occur by the direct action of gramnegative bacteria, or by indirect action, in which tissue destruction reactions are mediated by the host activating alternative pathways of inflammation [2].

The anatomical proximity of the bacterial biofilm to the periodontal vascular system facilitates the systemic dissemination of inflammatory bacteria and mediators, with the potential to develop complications for the organism. The relationship between periodontitis and other pathological conditions can be established by the immunogenic potential of host and / or bacterial products that reach the bloodstream and affect distant organs and systems. Data corroborating this idea are based on episodes in which some diseases, such as atherosclerosis, diabetes and rheumatoid arthritis, are associated with peridontopathy due to inflammatory and immunoregulatory mechanisms[2-4].

Human conditions, such as hospitalizations in Intensive Care Units (ICUs), the installation of diseases and injuries that lead to prolonged rest periods, as well as the immobilization

Address for correspondence: Gladson Ricardo Flor Bertolini, Universidade Estadual do Oeste do Paraná, Rua Universitária, 2069., 85819110, Cascavel, Brazil

E-mail: gladsonricardo@gmail.com

Received: 9 January 2019; accepted: 22 February 2019; first published: 21 March 2019 of a limb, are capable of promoting complications in the various systems of the organism, especially in the affecting musculature the muscular tissue, with functional and structural alterations such as the loss of muscular mass [5-7].

Loss of muscle mass due to disuse has been related to decreased synthesis and increased protein degradation [8], as well as the disruption of adjacent fibres and tissues that interfere with fibre length, weight and size because, consequently, there is interference in muscular functioning[9-11]. However, there are gaps in the literature regarding the action of these two associated conditions, since the muscle tissue responds to the stimuli to which they are submitted; when there is disuse there may be alterations in the shape of muscle fibres as well as processes that contribute to inflammatory damage in the muscle [12-13]. Since periodontal disease has systemic effects, there are correlates of muscle changes resulting from systemic inflammation $[5,14]$. Thus, the objective of this study was to evaluate whether periodontal disease, through systemic inflammation, potentiates the deleterious effects of immobilization on the anterior tibialis muscle of Wistar rats.

\section{MATERIALS AND METHOD}

The study was conducted according to the international norms of ethics in animal experimentation and approved by the Committee for Ethics in Animal Use of the State University of West of Paraná (UNIOESTE). The work was developed at the Laboratory for the Study of Injuries and Physiotherapeutic Resources (LELRF) and in the Laboratory 
of Structural and Functional Biology (LABEF), linked to the university.

The sample group consisted of 20 adult Wistar rats, kept in the LELRF 's spa area, under controlled conditions of temperature $\left(23 \pm 2^{\circ} \mathrm{C}\right)$ and light $(12$ - hour light cycle and $12 \mathrm{~h}$ dark cycle - 07:00 - 19:00), with water and commercial food ad libitum.

The animals were randomized into four groups $(n=05)$ : Control Group (CC), without any type of intervention, with the animals remaining free in the cage during the whole experiment; Periodontal Disease Group (PDG), with animals submitted to periodontal disease; Immobilization Group (IG): animals submitted only to immobilization of the right pelvic limb; and Periodontal Disease and Immobilization Group (PDIG): in which animals were submitted to periodontal disease and immobilized.

Periodontal disease and experimental immobilization. After a week of acclimatization, on the first day of the experiment the animals were anaesthetized with xylazine hydrochloride $(0.04 \mathrm{~mL} / 100 \mathrm{~g})$ and ketamine hydrochloride $(0.08 \mathrm{~mL} / 100 \mathrm{~g})$ intraperitoneally. Subsequently, they were placed on an appropriate operative table to maintain the mouth opening, facilitating access to the teeth in the posterior region of the mandible. With the aid of a modified forceps and an exploratory probe, a cotton thread 40 was inserted around the main right and left lower first molars. This ligature remained for 30 days as a gingival irritant, favouring the accumulation of bacterial plaque and consequent development of periodontal disease[15].

After 15 days of induction of periodontal disease, the animals were anaesthetized and immobilized, using tissuesaturated tissue dressing with dehydrated calcium sulfate (CaSO4) in the form of white powder, characterizing a cast bandage. For this, the immobilized experimental groups had the orthosis moulded from the abdominal region, just below the last ribs, following the right hind limb of each animal, with the bandage being placed throughout the limb extension, that is, in extension of the knee joint, as well as complete ankle plantar flexion in the position of stretching of the anterior tibial muscle. The animals were kept in this position for a period of 15 consecutive days[16].

Histological processing in anterior tibial muscle. On day 30 of the experiment, all animals were anaesthetized again, decapitated in a guillotine, and the right anterior tibial muscle collected for histomorphometric analysis. The muscle was fixed in Metacarn Solution and after 24 hours stored in 70\% alcohol. Subsequently, they were dehydrated in increasing alcoholic series, diaphanized, included and placed in histological paraffin. After obtaining the blocks, the material was cut transversely with the use of a rotating microtome, in the thickness of $5 \mu \mathrm{m}$. The obtained slides were stained in Haematoxylin and Eosin and analyzed for the cross-section area, the largest and smallest diameter and the number of cores. For these measurements, 10 images were obtained on the $40 \mathrm{X}$ objective, and in each image 10 fibres were measured by the Image-Pro Plus 6.0 program, totaling 100 fibres per animal. The nuclei present in the photomicrography were identified, marked and counted. The slides were also stained in Masson's Trichrome for quantification of connective tissue with the GIMP 2.0 programme.
Statistical analysis. Descriptive statistics of the data and evaluation of normality were performed with the ShapiroWilk test. For analysis of the variance uni-directional ANOVA was used, with LSD post-test ( $\mathrm{t}$ ); the level of significance was $5 \%$, for which the Bioestat 5.0 programme was used.

\section{RESULTS}

For the cross-sectional area $(\mathrm{F}=9.9036 ; \mathrm{p}=0.0012)$ and the largest diameter $(\mathrm{F}=3,668 ; \mathrm{p}<0,0343)$, there was a difference between the groups, denoting that the values of this variable were decreased in the immobilization process. For the smallest diameter, there was a significant difference $(\mathrm{F}=35.7003 ; \mathrm{p}<0.0001)$, indicating that the values of this variable were decreased when there was an association between periodontal disease and immobilization. There was a significant difference $(\mathrm{F}=16.7057 ; \mathrm{p}=0.0001)$ in the number of nuclei, indicating that there was an increase of nuclei with immobilization and its association with periodontitis. For the variable percentage of connective tissue, there was a significant difference $(\mathrm{F}=9.2183, \mathrm{p}=0.0012)$, with increase only when there was an association between immobilization and periodontitis (Tab. 1).

Table 1. Mean values of cross-sectional area, largest diameter, smaller diameter, in $\mu \mathrm{m}$, and percentage of connective tissue (\%) of the fibres of the anterior tibial muscle of Wistar rats

\begin{tabular}{lcccc}
\hline & CG & PDG & IG & DPIG \\
\hline Transverse Section & 1618.89 & 1472.61 & 1266.06 & 1125.86 \\
Area $\left(\mu \mathrm{m}^{2}\right)$ & $\pm 117.37^{\mathrm{A}}$ & $\pm 182.81^{\mathrm{AB}}$ & $\pm 190.06^{\mathrm{BC}}$ & $\pm 113.06^{\mathrm{C}}$ \\
\hline Largest diameter $(\mu \mathrm{m})$ & $52.13 \pm 1.61^{\mathrm{A}}$ & $\begin{array}{c}49.86 \\
\pm 4.42^{\mathrm{AC}}\end{array}$ & $\begin{array}{c}46.00 \\
\pm 4.66^{\mathrm{BC}}\end{array}$ & $\begin{array}{c}45.84 \\
\pm 2.75^{\mathrm{BC}}\end{array}$ \\
\hline Smallest diameter $(\mu \mathrm{m})$ & $34.11 \pm 0.87^{\mathrm{A}}$ & $31.57 \pm 1.16^{\mathrm{B}}$ & $29.88 \pm 1.37^{\mathrm{B}}$ & $26.87 \pm 1.06^{\mathrm{C}}$ \\
\hline Number of Nuclei & 480.80 & 511.20 & 683.40 & 884.2 \\
& $\pm 88.87^{\mathrm{A}}$ & $\pm 94.95^{\mathrm{AB}}$ & $\pm 132.32^{\mathrm{C}}$ & $\pm 83.39^{\mathrm{D}}$ \\
\hline Connective tissue $(\%)$ & $0.82 \pm 0.11^{\mathrm{A}}$ & $1.21 \pm 0.30^{\mathrm{A}}$ & $1.33 \pm 0.10^{\mathrm{A}}$ & $2.28 \pm 0.84^{\mathrm{B}}$ \\
\hline
\end{tabular}

CG - Control Group; PDG - Periodontal Disease Group; IG - Immobilization Group; DPIG Periodontal Disease Group + Immobilization. Values expressed as mean \pm standard deviation. Letters represent the statistically significant difference

Morphological analysis of anterior tibial muscle. The tibialis anterior muscle of the control group (CG) presented fibres with normal morphology and polygonal shape, multinucleate with nuclei in the peripheral position, and well-defined fascicles with organization and preserved structure. The intramuscular connective tissue of the endomysium and perimysium had a characteristic arrangement of the fibres (Fig. 1A).

In the group periodontal disease (GDP), the fibres also presented, in the majority, a similar appearance to the GC; however, there was a slight increase in the number of nuclei and some fibers had an irregular shape. Regarding the intramuscular connective tissue, the fibre arrangement was also similar to GC (Fig. 1B).

However, in the immobilized group (GI), the muscle fibres were atrophic, with a smaller diameter than in the GC and GDP groups, had an irregular shape and in some therec was nuclei degeneration in the central position. In addition, dislocation was observed in the intramuscular connective tissue (Fig. 1C).

The association of the two conditions, periodontal disease and immobilization (GDPI), potentiated atrophy and 


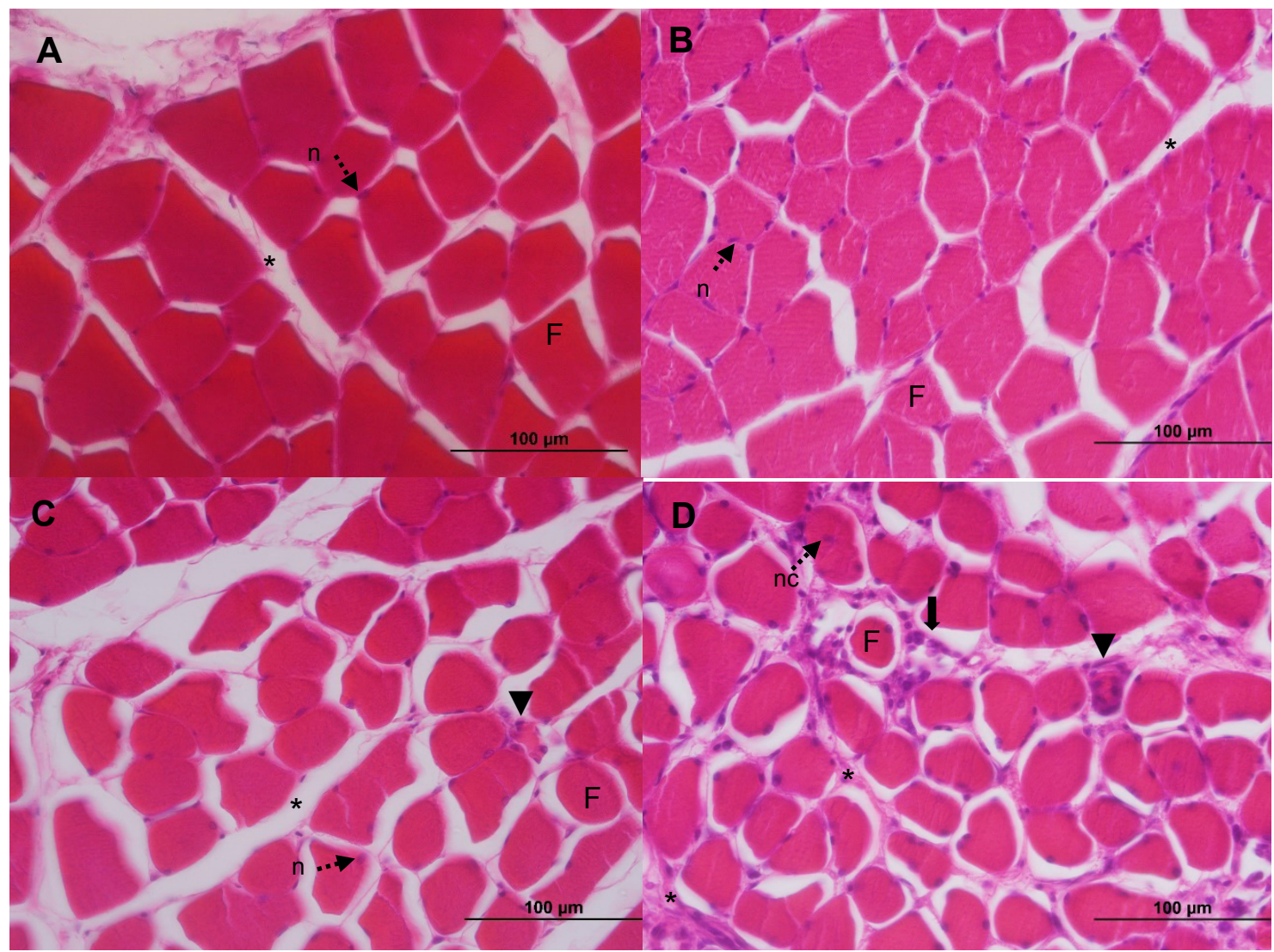

Figure 1. Photomicrographs of tibial anterior muscle of Wistar rats in cross-section with HE staining.

A - Control group, evidencing fibres of normal aspect with polygonal shape (F), peripheral nucleus (n), connective tissue with characteristic arrangement, perimysium (asterisk). B - Periodontal disease group, some fibres with irregular shape (F), increase of nucleus number. C - Immobilized group, showing atrophic and amorphous fibres $(F)$, and degenerating $(\boldsymbol{\nabla})$. D - periodontal and immobilized groups, atrophic and amorphous fibres $(F)$, and in degeneration ( $\mathbf{\nabla})$, centralized nodes (nc), inflammatory cells (black arrow) and conjunctive fibers increase in endomysium and perimysium (asterisks)

muscular degeneration, with the presence of a larger number of fibres with irregular and amorphous shape, increased nuclei and central position. A large number of inflammatory cells were observed in the interstitium, as well as changes in connective tissue (Fig. 1D).

\section{DISCUSSION}

The disuse of skeletal muscle alters muscle metabolism, leading to consequences of fibre alteration as well as functional change [17]. In the present study, it was possible to observe that the immobilization promoted reduction of the cross-sectional area of the muscle fibres, corroborating with the study by Slimani, et al.[18], who performed an immobilization model with tibialis muscle elongation, and found that after eight days of immobilization there was a reduction in the cross-sectional area due to the increase in muscle fibre proteolysis. A similar result was found by Kuntz et al. [16] in a model with 18 animals immobilized during 15 days in which a reduction of muscular mass observed. Identical findings were presented by Fujita et al. [29] in which suspension of the pelvic limb of rats resulted in muscle atrophy with reduction in the cross-sectional area.

Muscle fibers may alter physiological and biochemical properties according to the stimuli to which they are subjected. In immobilization, these changes can be explained because the maintenance of muscle mass depends on the homeostasis between protein synthesis and degradation, and during immobility there is a change in this balance, resulting in loss of muscle mass when the protein catabolic process exceeds the anabolic $[7,20]$.

Muscle atrophy can be explained by the ubiquitin proteasome pathway which is dependent on ATP, and is considered the main pathway of skeletal muscle degradation in response to disuse, being composed of E1, E2 and E3 ligases that regulate the degradation process. The main E3 ubiquitin is subdivided into MuRF1 and MAFbx that are found in striated muscle, and can be regulated by FOXO transcription factors which are involved in metabolism, apoptosis, and cell cycle progression, when FOXO is dephosphorylated to promote apoptosis $[21,22]$. Another possibility for the alterations found in immobilized groups is oxidative stress, since reactive oxygen species (ROS) play an important part in muscle homeostasis, but it can also cause muscle tissue loss. ROS and nitric oxide (NO) are capable of causing oxidative damage to DNA, lipids and proteins [23].

The association of immobilization with periodontal disease showed a reduction in the smaller diameter of the muscle fibre. In studies of Ramirez et al.[12, 13], when performing tibio-tarsal inflammation, there was an increase in the levels of MuRF-1 in the anterior tibial muscle, therefore, in the presence of inflammation, there was an increase of genes related to atrophy.

Regarding the cross-sectional area when periodontal disease was associated with immobilization, there was no reduction 
in the area, but there was reduction of the smallest diameter. This may have occurred because periodontal disease may induce a systemic inflammatory response, thus favouring the reduction of muscle fibre [5,24], which may have occurred due to synergy between oxidative stress, inflammatory cytokines and disuse, so that atrophy was accelerated or aggravated since, according to Fink et al. [25] immobilization and inflammation decrease muscle strength, with the combination of both factors becoming additive. In the present study, muscle strength was not compared, but changes in fibre diameter and increase in the number of nuclei, as well as the increase of inflammatory cells in the association of the two conditions, evidences the need to control diseases that lead to systemic inflammation when associated with immobilism.

Slimani et al. [18] observed that there was an increase in collagen expression in the tibialis immobilized in elongation, suggesting that the increase in its synthesis contributes to the increase of the connective tissue. The association of periodontal disease and immobilization showed an increase in connective tissue. Periodontal disease generates a systemic inflammation and, according to Hasturk et al.[26], if the host does not neutralize the pathogens, in the repair phase, fibrin is not efficiently eliminated after the acute phase of inflammation, with the formation of granulation tissue from the surrounding tissue compartments, thus resulting in the deposition of fibroblasts mediated collagen. The same was suggested by Taylor [27], because when there is a response to plaque, there occurs the activation of adaptive immune responses and the integration of immune regulation and connective tissue to promote homeostasis. This explainins the increase of collagen deposition in the tissue when both conditions were associated.

In this study, it was found that the association between periodontal disease and immobilization promoted potentiation of muscle fibre area reduction and augmentation of connective tissue. No studies were found relating these factors to the anterior tibial muscle; however, Leite et al. [5], in the same association model, found that there were morphological alterations of muscle fibres and an increase in the connective tissue of the soleus muscle, confirming that the association of both increases the harmful effects of immobilization in muscle tissue, regardless of the structure and molecular composition of the muscles.

\section{CONCLUSION}

With this study it is possible to conclude that periodontal disease potentiates the deleterious effects of immobilization in the anterior tibial muscle through the degeneration of muscle tissue, with reduction in the smaller diameter, increase in the number of nuclei, increase in connective tissue and presence of inflammatory infiltrate.

\section{REFERENCES}

1. Toledo GB, Cruz I. The importance of oral hygiene in Intensive Care Unit as a way of prevention of nosocomial infection - literature review. J Spec Nurs Care [Internet]. 2009;2(1). Available from: http://www. jsncare.uff.br/index.php/jsncare/article/view/j.1983-4152.2009.2047/453

2. Ramos MMB, Mendonça MR de, Pellizzer EP, Okamoto AC, Júnior EGJ. Associação entre a doença periodontal e doenças sistêmicas crônicas - revisão de literatura / Association between periodontal disease and chronic systemic disease - literature review. Arch Heal Invest. 2013;2(1): 24-31.
3. Fernandez-Solari J, Barrionuevo P, Mastronardi CA. Periodontal disease and its systemic associated diseases. Mediators Inflamm. 2015;2015: 153074.

4. Darveau RP, Tanner A, Page RC. The microbial challenge in periodontitis. Periodontol 2000. 1997; 14(79): 12-32.

5. Leite MA, de Mattia TM, Kakihata CMM, Bortolini BM, de Carli Rodrigues PH, Bertolini GRF, et al. Experimental periodontitis in the potentialization of the effects of immobilism in the skeletal striated muscle. Inflammation. 2017; 40(6): 2000-11.

6. França EÉT, Ferrari F, Fernandes P, Cavalcanti R, Duarte A, Martinez BP, et al. Physical therapy in critically ill adult patients: Recommendations from the Brazilian Association of Intensive Care Medicine Department of Physical Therapy. Rev Bras Ter Intensiva. 2012; 24(1): 6-22.

7. Ochala J, Gustafson AM, Diez ML, Renaud G, Li M, Aare S, et al. Preferential skeletal muscle myosin loss in response to mechanical silencing in a novel rat intensive care unit model: Underlying mechanisms. J Physiol. 2011; 589(8): 2007-26.

8. Jackman RW, Kandarian SC. The molecular basis of skeletal muscle atrophy. AJP Cell Physiol. 2004; 287(4): C834-43.

9. Piovesan RF, Fernandes KPS, Alves AN, Teixeira VP, Silva Junior JA, Martins MD, et al. Effect of nandrolone decanoate on skeletal muscle repair. Int J Sports Med. 2013; 34(1): 87-92.

10. Carvalho MM, Martins WR, Blackzick JC, Cruz Júnior CA da, Souza HA de, Kückelhaus SAS, et al. Análise morfométrica dos efeitos da imobilização sobre o músculo gastrocnêmio de ratos. Univ Ciências da Saúde. 2013; 11(2): 99-106.

11. Carvalho CMM de, Shimano AC, Volpon JB. Efeitos da imobilização e do exercício físico em algumas propriedades mecânicas do músculo esquelético. Rev Bras Eng Biomédica. 2002; 18(2): 65-73.

12. Ramírez C, Russo TL, Delfino G, Peviani SM, Alcântara C, Salvini TF. Effect of tibiotarsal joint inflammation on gene expression and cross-sectional area in rat soleus muscle. Brazilian J Phys Ther. 2013; 17(3): 244-54.

13. Ramírez C, Russo T, Sandoval M, Abbade Dentillo A, Abreu Silca Couto M, Quagliotti Durigan J, et al. Joint inflammation alters gene and protein expression and leads to atrophy in the tibialis anterior muscle in rats. Am J Phys Med Rehabil. 2011; 90(11): 930-9.

14. Winkelman C. Inactivity and inflammation: selected cytokines as biologic mediators in muscle dysfunction during critical illness. AACN Clin Issues. 2004; 15(1): 74-82.

15. Nassar PO, Nassar CA, Guimarães MR, Aquino SG, Andia DC, Muscara $\mathrm{MN}$, et al. Simvastatin therapy in cyclosporine A-induced alveolar bone loss in rats. J Periodontal Res. 2009; 44(4): 479-88.

16. Kunz RI, Coradini JG, Silva LI, Bertolini GRF, Brancalhão RMC, Ribeiro LFC. Effects of immobilization and remobilization on the ankle joint in Wistar rats. Brazilian J Med Biol Res. 2014; 47(10): 842-9.

17. Mallinson JE, Murton AJ. Mechanisms responsible for disuse muscle atrophy: Potential role of protein provision and exercise as countermeasures. Nutrition. 2013; 29(1): 22-8.

18. Slimani L, Vazeille E, Deval C, Meunier B, Polge C, Dardevet D, et al. The delayed recovery of the remobilized rat tibialis anterior muscle reflects a defect in proliferative and terminal differentiation that impairs early regenerative processes. J Cachexia Sarcopenia Muscle. 2015; 6(1): 73-83.

19. Fujita N, Murakami S, Arakawa T, Miki A, Fujino H. The combined effect of electrical stimulation and resistance isometric contraction on muscle atrophy in rat tibialis anterior muscle. Bosn J Basic Med Sci. 2011; 11(2): 74-9.

20. Bodine SC. Disuse-induced muscle wasting. Int J Biochem Cell Biol. 2013; 45(10): 2200-8.

21. Brooks NE, Myburgh KH. Skeletal muscle wasting with disuse atrophy is multi-dimensional: The response and interaction of myonuclei, satellite cells and signaling pathways. Front Physiol. 2014; 5(99): 10.3389.

22. Bodine SC, Baehr LM. Skeletal muscle atrophy and the E3 ubiquitin ligases MuRF1 and MAFbx/atrogin-1. Am J Physiol Endocrinol Metab. 2014; 307(6): E469-84.

23. Pellegrino MA, Desaphy JF, Brocca L, Pierno S, Camerino DC, Bottinelli R. Redox homeostasis, oxidative stress and disuse muscle atrophy. J Physiol. 2011; 589(9): 2147-60.

24. Oliveira JAP, Hoppe CB, Gomes MS, Grecca FS, Haas AN. Periodontal disease as a risk indicator for poor physical fitness: A cross-sectional observational study. J Periodontol. 2015; 86(1): 44-52.

25. Fink H, Helming M, Unterbuchner C, Lenz A, Neff F, Martyn JAJ, et al. Systemic inflammatory response syndrome increases immobilityinduced neuromuscular weakness. Crit Care Med. 2008; 36(3): 910-6.

26. Hasturk H, Kantarci A, Van Dyke TE. Oral inflammatory diseases and systemic inflammation: Role of the macrophage. Front Immunol. 2012; 3(118): 10.3389 .

27. Taylor JJ. Protein biomarkers of periodontitis in saliva. ISRN Inflamm. 2014; 2014: 593151. 